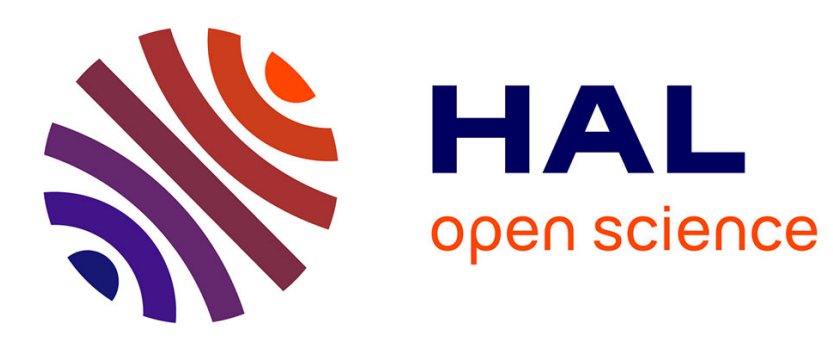

\title{
Investigating the kinetics of paramagnetic-beads linked alkaline phosphatase enzyme through microchannel resistance measurement in dielectric microchip.
}

\author{
Mathilde Faure, Bruno Sotta, Jean Gamby
}

\section{- To cite this version:}

Mathilde Faure, Bruno Sotta, Jean Gamby. Investigating the kinetics of paramagnetic-beads linked alkaline phosphatase enzyme through microchannel resistance measurement in dielectric microchip.. Biosensors and Bioelectronics, 2014, 58, pp.61-67. 10.1016/j.bios.2014.02.036 . hal-01011983

\section{HAL Id: hal-01011983 \\ https://hal.sorbonne-universite.fr/hal-01011983}

Submitted on 20 Oct 2014

HAL is a multi-disciplinary open access archive for the deposit and dissemination of scientific research documents, whether they are published or not. The documents may come from teaching and research institutions in France or abroad, or from public or private research centers.
L'archive ouverte pluridisciplinaire HAL, est destinée au dépôt et à la diffusion de documents scientifiques de niveau recherche, publiés ou non, émanant des établissements d'enseignement et de recherche français ou étrangers, des laboratoires publics ou privés. 


\title{
Investigating the kinetics of paramagnetic-beads linked alkaline phosphatase enzyme through microchannel resistance measurement in dielectric microchip
}

\author{
Mathilde Faure ${ }^{\mathrm{a}, \mathrm{b}}$, Bruno Sotta ${ }^{\mathrm{c}}$, Jean Gamby ${ }^{\mathrm{a}, \mathrm{b}, *}$ \\ ${ }^{a}$ CNRS, UMR 8235, LISE, F-75005, Paris, France \\ ${ }^{b}$ Sorbonne Universités, UPMC Univ Paris 06, UMR 8235, Laboratoire Interfaces et Systèmes \\ Electrochimiques, F-75005, Paris, France \\ ${ }^{c}$ Sorbonne Universités, UPMC Univ Paris 06, UMR 7622, Laboratoire Biologie des Semences, 4 Place \\ Jussieu, F-75005 Paris, France
}

* Corresponding author: jean.gamby@upmc.fr Tel: +331 442736 40; Fax: +33 14427 4074; 


\begin{abstract}
Real time monitoring of electrolyte resistance changes during hydrolysis of 4nitrophenylphosphate (pNPP) by alkaline phosphatase (ALP) bound on paramagnetic-beads was performed into a small dielectric channel. The reaction kinetic fit with a non-competitive substrate-inhibition equation. Michaelis-Menten apparent constant, $K_{M}^{a p p}$, was determined as $0.33 \pm 0.06 \mathrm{mM}$ and the maximum apparent rate, $V_{\max }^{a p p}$ as $98 \pm 5 \mathrm{pM} \mathrm{s}^{-1}$. The detection limits were $15 \mathrm{fM}$ for ALP and $0.75 \mathrm{mM}$ for $\mathrm{pNPP}$. This miniaturized device constitutes a powerful tool for analysis of interaction between ligands.
\end{abstract}

Keywords: dielectric microchannel; electric impedance; paramagnetic-beads; alkaline phosphatase; 4-nitrophenylphosphate; enzyme kinetics. 


\section{Introduction}

The development of miniaturized total chemical analysis systems ( $\mu$ TAS) has been boosted, in a biotechnological point of view, due to the advantage of microdevices $[1,2,3,4]$. In small devices experiments can be quickly performed in pico volume, reaping advantages from the high surface/ volume ratio in microchannels $[5,6,7]$. In order to obtain low detection limits microdevices need highly sensitive techniques. Until now optical $[8,9,10]$ or electrochemical $[11,12,13]$ techniques have been preferably integrated in microdevices for detection. For two decades detectors in a non contact configuration have known a renewed interest in capillary electrophoresis (CE) for the detection of ions or biomolecules [14,15]. After the separation step ions or biomolecules can be detected through tubular electrodes by applying a high-frequency alternative signal. The detected peaks are related to a change of conductivity between the two electrodes as soon as the migration of species takes place. In previous papers $[16,17,18]$ the concept of contact free detection has been revisited by making directly well defined microchannels and embedded microelectrodes in an insulated polymer by using laser photoablation. This process offers a very good resolution with an optimal position of microelectrodes. Contact free detection in a microchip limits electric coupling, bubbles generation, microelectrode passivation as well as any contamination, and degradation of the enzyme due to uncontrolled faradic reactions. The interface constituted by a thin polymer layer sandwiched between the two planar microelectrodes and the microchannel can be considered as a dielectric [19], and its properties can be easily characterized by impedance techniques. Thus, a detailed methodology for non-contact impedance measurement in microchips was proposed taking into account, on the measurement accuracy, the role played by the

surrounding polymer [20]. Measurements were carried out using polyethylene terephthalate (PET), which is a well-known dielectric polymer. The procedure described previously demonstrated the efficiency of the elimination step of the PET impedance contribution on the 
global microchip impedance [20,21]. This operation enabled a clear observation of the impedance in the microchannel solution such as the bulk solution contribution and the interfacial capacitance related to the surface roughness of the photoablated microchannel.

The purpose of the work depicted below is, on the one hand, a significant simplification of the previous approach methodology [20]. Indeed, the originality of the present approach is that the signal can be analyzed from differential measurement acquisitions of the resistive component of the electric impedance measured through the dielectric microchip, linked to microchannel resistance changes. Briefly, this new approach permits to record in time the resistance of a microchannel in a dielectric microchip and is illustrated with detailed kinetic parameters of the hydrolysis of 4-nitrophenylphosphate (pNPP) into 4-nitrophenol (pNP) by alkaline phosphatase (ALP). It represents a substantial improvement of the simplified methodology without the use of chromophogenic or electroactive properties of enzymatic substrates [22,23,24]. In order to reuse the same PET microchannel after several series of runs, the loading of superparamagnetic microbeads coated ALP into the small channel was preferred for limiting non specific biological adsorption on PET surface and for offering a high ratio of reactive surface/volume. The use of a permanent magnet enables the aggregation of the microbeads in the microchannel within the detection area between the two planar embedded microelectrodes [21,25].

\section{Experimental}

\subsection{Reagents and chemicals}

Magnesium chloride, Sodium chloride, Tris(hydroxymethyl)aminomethane (Tris) and 4nitrophenylphosphate (pNPP) were purchased from Sigma Aldrich. 2-methylaminoethanol (MAE) was received from Fluka Analytical. The washing buffer was prepared with 0.1 M Tris and $0.5 \mathrm{M} \mathrm{NaCl}, \mathrm{pH}$ 9. The substrate buffer was prepared with $0.1 \mathrm{M}$ MAE and $0.002 \mathrm{M} \mathrm{MgCl}_{2}$. Deionized and double-distilled water was used as regeneration solution. Alkaline Phosphatase at 
$3 \mathrm{mg} \mathrm{mL}^{-1}$ was from intestine of veal (Boehringer, Mannhein). The stock solution of microbeads (Dynabeads $^{\mathrm{R}}$ ) was purchased from Invitrogen France. The microbeads concentration was equal to $10 \mathrm{mg} \mathrm{mL}^{-1}$ with of $4 \times 10^{8}$ microbeads per milliliter in phosphate buffer saline (PBS, $\mathrm{pH} 7.4$ ) containing $0.1 \% \mathrm{BSA}$ and $0.02 \% \mathrm{NaN}_{3}$ as preservative.

\subsection{Sample preparation}

Superparamagnetic-beads $(160 \mu \mathrm{L})$ were washed three times prior to use with $1 \mathrm{~mL}$ Tris. The washed solution of microbeads was adjusted with fresh washing buffer to obtain a final concentration of $0.2 \mathrm{mg} \mathrm{mL}^{-1}$. ALP was coupled with avidin according to Avrameas et al. [26]. The stock solution of ALP-avidin was equal to $4.6 \mu \mathrm{g} \mathrm{mL}^{-1}$. Six samples of ALP-avidin (ALP-av) coupled with $1 \mu \mathrm{m}$-microbeads ( $\mu$ Beads) were prepared following successive dilutions according to proportions described in Table S1 (see supplementary information).

\subsection{Microelectrodes and microchannel Networks}

Procedures of photoablated microchannel have been described in previous papers [16,27]. Briefly, a $100 \mu \mathrm{m}$ thick polyethylene terephthalate sheet was photoablated using a $193 \mathrm{~nm}$ argonfluor excimer laser (Lambda Physik LPX 2051) in order to obtain a $2 \mathrm{~cm}$ long with a $45 \mu \mathrm{m}$ depth trapezoidal microchannel, with $100 \mu \mathrm{m}$ and $80 \mu \mathrm{m}$ top and bottom widths (see Fig. 1A). On the other side of the PET film two parallel microchannels, with a depth of $50 \mu \mathrm{m}$ and a width of $100 \mu \mathrm{m}$, were photoablated perpendicularly to the surface (see Fig. 1B). As a consequence the non contact layer is equal to $5 \mu \mathrm{m}$ (Figs. 1A-B). The gap between these two parallel microchannels was equal to $200 \mu \mathrm{m}$ center to center (see Figs. 1A-B). The two channels were then filled with a commercial graphite ink (Electra $\Omega$ ED5000 Electra Polymers). Another film of 
PET/PE $(35 \mu \mathrm{m})$ was laminated on the face of the PET film including the two carbon electrodes by heating at $60{ }^{\circ} \mathrm{C}$ for $4 \mathrm{~h}$ (Fig. 1B). The microelectrodes were connected to wires by a silver conducting paste. The total volume of the microchannel was $80 \mathrm{~nL}$.

\subsection{Apparatus}

Electric impedance measurements were performed on a Dielectric interface Solartron 1260 gain phase impedance analyzer coupled with a frequency response analyser (FRA 1255) and computer-controlled (Smart software, Solartron Analytical). The frequency range of electric impedance measurements was from $20 \mathrm{~Hz}$ to $20 \mathrm{MHz}$. The current measurement range of the system was from $6 \mathrm{~mA}$ to $100 \mathrm{fA}$, thus enabling the measurements of very high impedance up to $100 \mathrm{~T} \Omega$. The microchip was connected to a dielectric interface apparatus. In order to follow bioanalytical application at a fixed frequency a high-frequency window has been preferred because PET behaves as a pure capacitor (see Fig. 1B) and the signal background is not disturbed by stray resistances in PET layers which are predominant at low frequencies. The main information contained in the real part of impedance, $\Re(Z)$, is the solution microchannel resistance, $R_{\mathrm{S}}$ and, on a technical point of view, signal time acquisition for measurement is the fastest at high frequencies.

\subsection{Methodology for electric impedance measurements in microchannel}

A voltage was applied between the two microelectrodes and the amplitude and the phase shift of the resulting current were measured. The ratio of the output signal to the input perturbation, called the transfer function, gave the global impedance $Z_{g} . Z_{g}$ is a complex number expressed as follows, 
$Z_{g}=\mathfrak{R e}\left(Z_{g}\right)+j \mathfrak{I} m\left(Z_{g}\right)$

where the resistance, $\Re e\left(Z_{g}\right)$, and the reactance, $\mathfrak{I} m\left(Z_{g}\right)$, are the real and imaginary components of impedance, respectively.

The characteristics of this kind of interface with an insulated PET layer sandwiched between two galvanic isolated microelectrodes can be represented as a dielectric system with, on one part of the thin polymer layer, electronic charges on microelectrodes and, on the other part, ionic charges in the microchannel considering impedances for each part of the microchip as described in a previous paper [20].

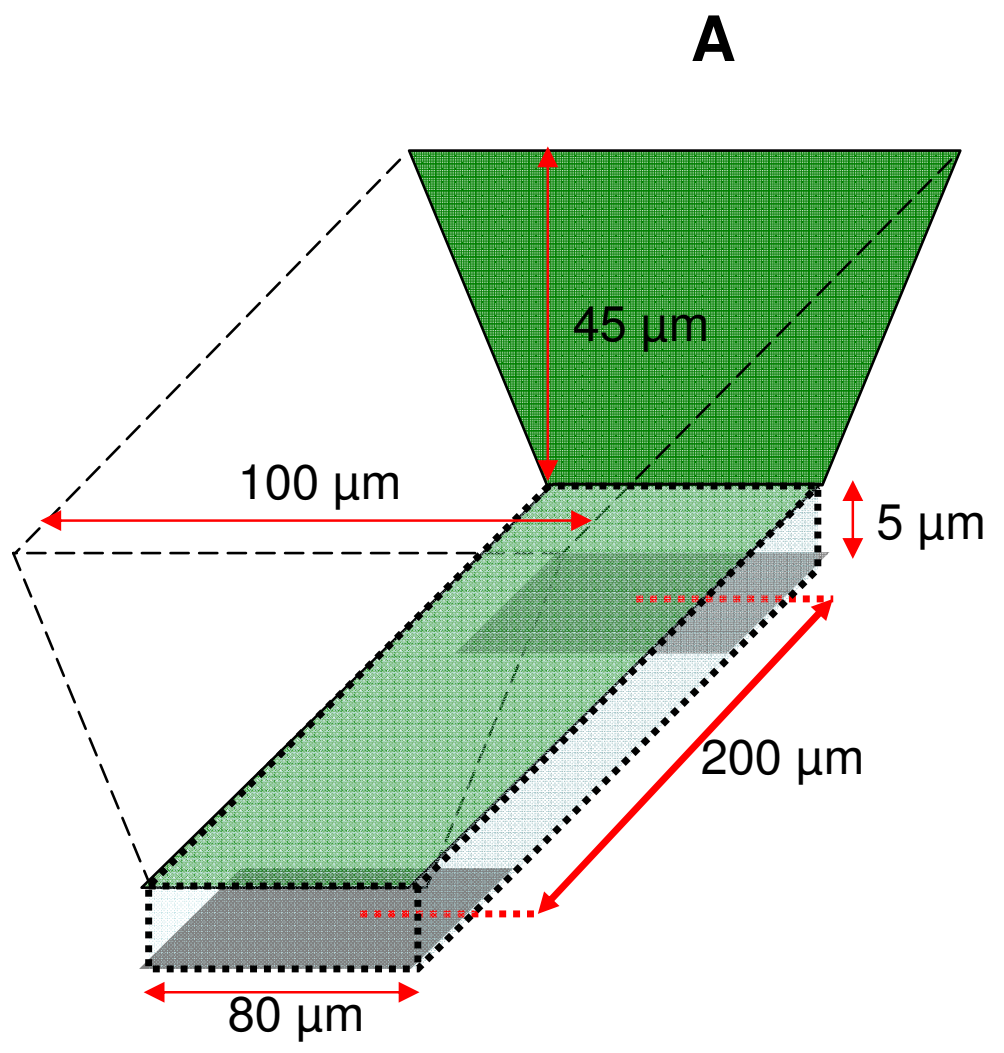




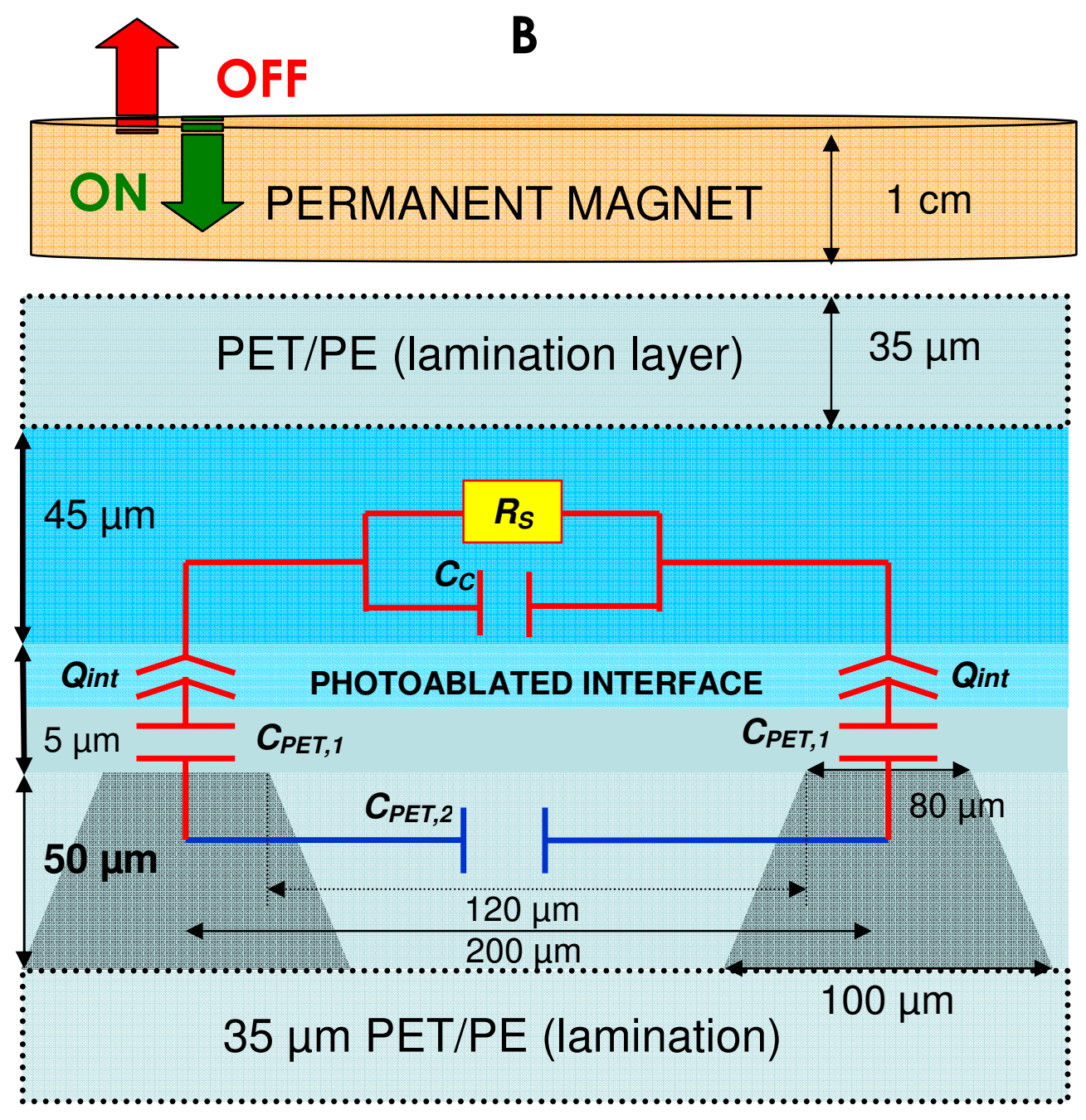

Fig. 1. Schematic view of the dielectric interface microelectrodes/PET /microchannel. (A) Trapezoidal PET microchannel with the two planar embedded microelectrodes without contact. (B) Cross section of the dielectric interface microelectrode/PET/ microchannel and the corresponding electrical equivalent circuit: $C_{\mathrm{PET}, 2}$ for the $120 \mu \mathrm{m}$-PET layer impedance (distance separation), $C_{\mathrm{PET}, 1}$ for the $5 \mu \mathrm{m}$-PET layer impedance (thickness of PET without contact layer), the element CPE, $Q_{\text {int }}$, for the interfacial impedance (photoablated surface), and the parallel association of the solution resistance, $R_{\mathrm{S}}$, and the cell capacitance, $C_{\mathrm{C}}$. Please note that for a more detailed view of the interface the dimensions are not to scale. 
As displayed in Fig. 1B the global impedance, $Z_{g},(\omega)$, measured through the dielectric microdevice contains two contributions. Indeed, it can be viewed as a parallel association between the current streamlines passing through the $5 \mu \mathrm{m}$-PET dielectric non contacting layer until the PET microchannel (red line in Fig. 1B), corresponding to the impedance, $Z_{1}(\omega)$, and the $120 \mu \mathrm{m}$-PET dielectric separating layer (blue line in Fig. 1B) corresponding to the impedance, $Z_{2}$ ( $\omega)$. Indeed, the global impedance can be expressed as a combination of the two impedances (see Eq. 2).

$\frac{1}{Z_{g}(\omega)}=\frac{1}{Z_{1}(\omega)}+\frac{1}{Z_{2}(\omega)}$

wherein is the angular frequency $\left(\operatorname{rad~s}^{-1}\right)$, defined by $\omega=2 \pi \mathrm{f}$, where $\mathrm{f}$ is the frequency $(\mathrm{Hz})$. The impedances $Z_{\mathrm{g}}(\omega)$ and $Z_{2}(\omega)$ were obtained from the measurement of impedance in the microdevice with the filled and empty microchannel, respectively. In order to determine the impedance $Z_{2}(\omega)$, the voltage was applied across the empty microchannel between two conductive planar carbon microelectrodes galvanically isolated in PET. The reproducibility of the obtained data was verified for each PET microchip before all experiments. In all case, the impedance $Z_{2}(\omega)$ written in Eq. 3 was found to a pure capacitive behavior and was estimated around $C_{\mathrm{PET}, 2}=8 \times 10^{-13}(\mathrm{~F})$.

$$
Z_{2, P E T}(\omega)=\frac{1}{j \omega C_{2, P E T}}
$$

The acquisition and interpretation of electric impedance are achieved with full microchannel (Fig. 1B). For that, the impedance $Z_{1}(\omega)$ written in Eq. 4 was represented by the series association of the impedance of the $5 \mu \mathrm{m}$-PET layer, the impedance of the PET/microchannel interface and the impedance of the solution, as follows: 


$$
Z_{1}(\omega)=2 Z_{1, P E T}(\omega)+2 Z_{\text {int }}(\omega)+Z_{\text {microchannel }}(\omega)
$$

with:

$$
\begin{aligned}
& Z_{1, \text { PET }}(\omega)=\frac{1}{j \omega C_{1, P E T}} \\
& Z_{\text {int }}(\omega)=\frac{1}{(j \omega)^{\alpha_{\text {int }}} Q_{\text {int }}} \\
& Z_{\text {microchannel }}(\omega)=\frac{R_{S}}{1+j \omega C_{C} R_{S}}
\end{aligned}
$$

The impedance of the $5 \mu \mathrm{m}$-PET written in equation 5 can be also represented by a pure capacitive behavior and was estimated around $C_{\mathrm{PET}, 1}=14 \times 10^{-11} \mathrm{~F}$. The impedance of the PET/microchannel interface was analyzed with a CPE behavior (Equation. 6) where its exponent, $\alpha_{i n t}$, and the CPE element, $Q_{i n t}$, were found equal to 0.5 and $4.7 \times 10^{-8} \Omega^{-1} \mathrm{~s}^{-\alpha}$, respectively. This typical value for $\alpha_{\text {int }}$ was attributed to the surface roughness obtained after the laser photoablation process [20]. The impedance for the microchannel solution was represented in equation 6 by taking into account the parallel association of the solution resistance, $R_{\mathrm{S}}$, and the cell capacitance, $C_{\mathrm{C}}$, which corresponds to the geometrical capacitance. This latter was equal to $C_{\mathrm{C}}=3 \times 10^{-13} \mathrm{~F}$. [20].

The target application is the real-time monitoring of the real part of the impedance variation of $\Delta \Re$ e $\left(Z_{\mathrm{g}}\right)$ which reflects mainly the electrolyte composition change in the microchannel (see Eqs 2, 4 and 7). To this aim, only the real part of the electric impedance which contains its resistive contribution has been taken into account. In the case of relative variation of the electric impedance in the microchip, the following simplifications were assumed. The interfacial 
impedance, $Z_{\text {int }}$ is known and fixed because no modification on the photoablated PET surface occurs throughout the course of the experiment. The $5 \mu \mathrm{m}$-PET layer impedance from the top of one electrode to the channel bottom is fixed and will be suppressed during the relative real part measurements. The same assumption can be done for the $120 \mu \mathrm{m}$-PET layer impedance between the two galvanically isolated microelectrodes. As a consequence, the real-time monitoring of the microchannel resistance can be measured through the relative variation, $\Delta \Re \mathrm{R}\left(Z_{\mathrm{g}}\right)$. Indeed, this latter at high frequencies mainly reflects the contribution of the microchannel resistance as follows:

$$
\Delta \Re e\left(Z_{g}\right) \cong \Delta R_{S}
$$

\subsection{Methodology for measurement enzymatic kinetics rate in a microchannel}

As reported in our previous paper [20], a small excitation of interface at high frequencies leads to direct information about the equivalent electrical circuit of the interface. In the present paper, the procedure has been simplified by measuring the real component of the impedance in an electric impedance measurement approach, since it is performed automatically with a $3 \mathrm{~V}_{\mathrm{pp}}$ excitation signal at a $1 \mathrm{MHz}$ fixed frequency. This configuration allows a simplification for the data processing in real time and also a fast acquisition time. Additionally, the present study investigates the detailed kinetics of ALP catalyzed pNPP hydrolysis. Samples are introduced by capillarity in the PET microchannel with a total volume capacity of $80 \mathrm{~nL}$ due to its trapezoidal cross section.

For each step of the detection procedure the real part of the electric impedance curve, $\mathfrak{R e}(Z \mathrm{~g})$, was recorded at $1 \mathrm{MHz}$ fixed frequency against time until a stationary signal as a baseline 
between each step. During the hydrolysis the real part of the impedance was sequentially measured versus incubation time without flow (see equation 9).

$d \frac{\left\lfloor\Delta \Re e\left(Z_{g}\right)_{1 M H z}\right\rfloor}{d t}=\frac{d \Delta R_{S}}{d t}$

As displayed in Fig. 1B a permanent magnet located above the detection zone was switched on the position ON. In brief, monitoring experiments of ALP catalyzed hydrolysis of an enzymatic substrate was done in three steps. First, the real part of the electric impedance for a microchannel filled with the substrate buffer was measured. Second, the microchannel was filled with an ALPavidin coupled with $\mu$ beads sample (see Table S1 in supplementary information) and a measurement protocol similar to that described in the first step was followed until a stationary signal was reached. The third step consisted in loading the reservoir with the enzymatic substrate pNPP.

\section{Results and discussion}

In order to determine the catalytic constant of the enzyme activity of ALP with pNPP substrate, the procedure described above was followed for different concentrations of ALP. The experimental data consist, for each enzyme substrate concentration, in a set of $R_{\mathrm{S}}$ experimental curves that are recorded as a function of the measurement time, $t$. Briefly, after at least $20 \mathrm{~s}$ of incubation time following the introduction of the enzymatic substrate a clear increase of $R_{\mathrm{S}}$ was observed. For that purpose, $\Delta R_{\mathrm{S}}$ variation against time during catalytic hydrolysis of $\mathrm{pNPP}$ was plotted from typical $R_{\mathrm{S}}=f(\mathrm{t})$, after normalization by subtracting the background signal at $t_{0}$. Eventually, the initial rate, $v_{i}$-slope, of $\Delta R_{\mathrm{S}}$ variation with time (see Eq. 10) gives information about the following kinetics involved and related to ALP and pNPP concentration, respectively. 
$v_{i}=\frac{d \Delta R_{S}}{d t} \quad\left(\right.$ in $\left.\Omega \mathrm{s}^{-1}\right)$

These observations established that $\Delta R_{\mathrm{S}}$ variation with time was due to the ionic charge changes in microchannel solution. This variation was clearly explained by a change in the resistivity of the microchannel solution related to the conductivity. Indeed, when pNPP was converted into $\mathrm{pNP}$ and phosphate ion, the number of charge carriers changed in the detection zone.

The kinetic rate constants, determined by the means of the above procedure, were considered as pseudo-first-order rate constants due to low enzyme concentration (pM range) in comparison to high enzymatic substrate concentration ( $\mathrm{mM}$ range). The $v_{i}$-slope values were plotted against the enzyme concentration giving rise to a straight line, as written in Eq. 10, with a slope that corresponded to the hydrolysis catalytic second-order rate constant, $k_{C A T}$, and $k_{0}$ for first-order hydrolysis determined without enzyme added.

$v_{i}=k_{C A T}[A L P]+k_{0}$

with $k_{C A T}$ in $\Omega \mathrm{s}^{-1} \mathrm{M}^{-1}$ and $k_{0}$ in $\Omega \mathrm{s}^{-1}$.

In order to determine these parameters, impedance curves for various enzyme concentrations (from $0.015 \mathrm{pM}$ to $1.21 \mathrm{pM}$ ) against fixed pNPP concentration (12 mM) versus incubation time were conducted (see Fig. 2). 


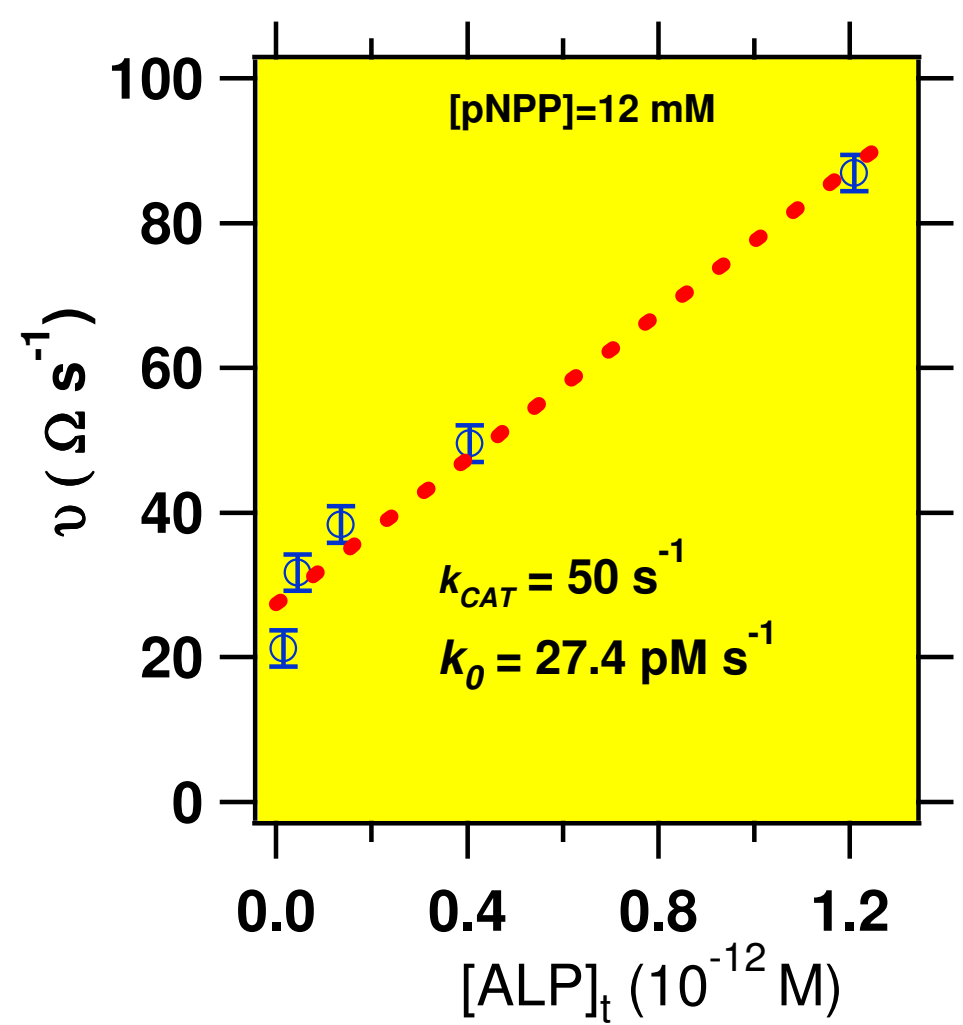

Fig. 2: Determination of hydrolysis catalytic rate constant, $k_{\mathrm{CAT}}$, from the calibration curve. The maximum rate, $v_{\mathrm{i}}$-slope, is plotted against ALP concentration with a fixed $12 \mathrm{mM}$ pNPP concentration. Each point represents the mean and S.D. (cross-hatched bars) of experiments with ALP concentration (from $0.015 \mathrm{pM}$ to $1.21 \mathrm{pM}$ ). The linear fit (dashed line) was performed according to Eq. 11 (in the text).

The units in $\Omega \mathrm{s}^{-1} \mathrm{M}^{-1}$ and $\Omega \mathrm{s}^{-1}$ were converted in more standard units $i . e . \mathrm{s}^{-1}$ and $\mathrm{M} \mathrm{s}^{-1}$, respectively, by taking in account the method sensitivity $\left(1 \Omega \mathrm{pM}^{-1}\right)$. This latter was determined from the calibration curves displayed in Fig. 2. The obtained values in standard units for $k_{C A T}$ and $k_{0}$, determined by a linear fit as expressed in Eq. 11 , were equal to $50 \pm 5 \mathrm{~s}^{-1}$ and $27.4 \pm 2.8 \mathrm{pM}$ $\mathrm{s}^{-1}$, respectively. The ALP calibration and the values of $k_{C A T}, k_{0}$ (with $12 \mathrm{mM}$ pNPP enzymatic substrate) are presented in Fig. 2. 
The Michaelis-Menten kinetic approach can be used in order to verify the validity of the kinetic parameters determination using these current method and methodology. These well-known kinetic parameters are the Michaelis-Menten constant, $K_{M}$, and the maximum rate, $V_{\max }$, for ALP with various pNPP concentration as enzymatic substrate. Typical $\Delta R_{\mathrm{S}}$ plotted against time, displayed in Fig. S1 (see supplementary information), illustrate data obtained for an increase of enzyme substrate concentration when the procedure described above was followed. The hydrolysis rate, $v_{i}$ is represented by the slope of the $\Delta R_{\mathrm{S}}$ curve that is linear for at least $20 \mathrm{~s}$ after the beginning of hydrolysis. When the quantity of the added enzyme substrate pNPP increases, $v_{i}$ increases until reaching a maximum rate, $V_{\max }$, which is a consequence of ALP sites saturation. ALP concentrations $\left(10^{-12}-10^{-15} \mathrm{M}\right)$ remained always negligible compared to pNPP concentration (millimolar range). This latter condition allows the use of Michaelis-Menten kinetic approach that is widely used due to its commodity. Briefly, ALP and pNPP react to form a fast equilibrium, with a corresponding constant, $K$, as shown in scheme $1 \mathrm{~A}$. The activated complex, $[A L P-p N P P]^{\neq}$, resulting from this equilibrium, decays slowly giving the corresponding alcohol, pNP, the phosphate ion, $\mathrm{PO}_{4}^{3-}$, and the regenerated ALP.

A

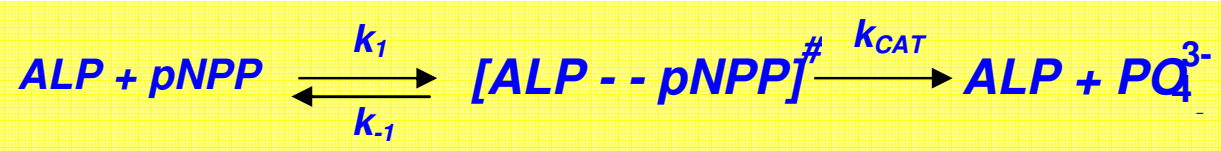

$$
\begin{gathered}
{[p N P P-A L P=-p N P P]^{\#}} \\
\qquad L P+p N P P \underset{k^{\prime}}{\stackrel{k_{1}}{\rightleftarrows}}[A L P=-p N P P]^{\stackrel{k_{C A T}}{\longrightarrow}} A L P+P Q_{4}^{3-}
\end{gathered}
$$


Scheme 1. (A) Reaction scheme of pNPP hydrolysis by ALP in alkaline media ( $\mathrm{pH}=9$ ). (B) Kinetic scheme of ALP hydrolyzed pNPP with substrate in excess i.e. "non competitive" inhibition.

As displayed in scheme $1 \mathrm{~A}$, the equilibrium constant, $K$, is a ratio of two kinetic constants, $K=k_{1} / k_{-1}$. The last step constitutes the rate-determining enzymatic step as expressed in the following Eq. 12 and the assumption that the activated complex concentration remained constant during time experiment (quasi-steady state hypothesis) as written in Eq. 13.

$$
\begin{aligned}
& v=\frac{d\left[P O_{4}^{3-}\right]}{d t}=k_{c a t}[A L P-p N P P]^{\neq} \\
& v=\frac{d[A L P-p N P P]^{\neq}}{d t}=0
\end{aligned}
$$

After simplification using conditions about initial concentrations of pNPP and ALP, i.e. ALP initial concentration remains negligible compared to pNPP initial concentration, and about total ALP concentration (free ALP and bound ALP) that doesn't vary over time experiment, and about mass balance expression for each species, the kinetic rate can be simplified as:

$-\frac{d[p N P P]}{d t}=\frac{V_{\max }[p N P P]}{K_{M}+[p N P P]}$

where $V_{\max }=k_{c a t}[A L P]_{t o t}$ is defined as the maximum rate and, $K_{M}=\frac{k_{-1}+k_{2}}{k_{1}}$, represents the Michaelis-Menten constant.

To determine these parameters an isotherm adsorption was plotted in Fig. 3 from $\Delta R_{\mathrm{S}}$ with time at fixed concentration of enzyme (i.e $0.135 \mathrm{pM}$ ) against pNPP concentration (from 0.25 to 12 $\mathrm{mM}$ ) versus incubation time (see Fig. 1S). Values for $K_{M}$ and $V_{\max }$ were determined by the 
fitting procedure using the adsorption isotherm (Eq. 14), and were found equal to $0.25 \mathrm{mM}$ and $41.3 \mathrm{pM} \mathrm{s}^{-1}$, respectively.

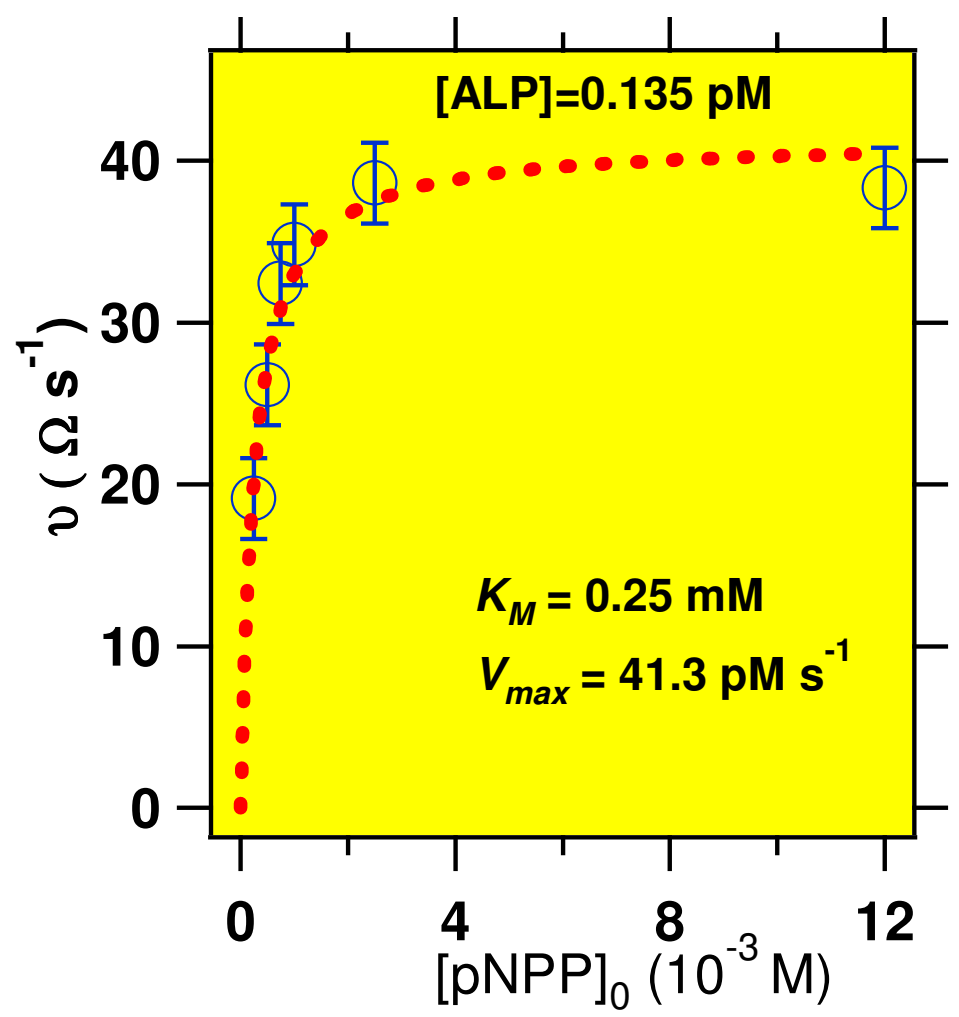

Fig. 3: Determination of Michaelis-Menten parameters, $V_{\max }$ and $K_{\mathrm{M}}$, from the isotherm adsorption plot. The initial rate, $v_{\mathrm{i}}$-slope, was plotted against $\mathrm{pNPP}$ concentration with a fixed 0.135 pM ALP concentration. Each point represents mean and S.D. (cross-hatched bars) of six experiments with pNPP concentration from $0.25 \mathrm{mM}$ to $12 \mathrm{mM}$ (See Fig. $\mathrm{S} 1$ in the supplementary information). The nonlinear regression (dashed line) was performed using the full Michaelis-Menten equation (see Eq. 14 in the text).

On close inspection on the fitting results of Fig. 3, it can be noted a deviation between the experimental isotherm and the theoretical one, when pNPP concentration is high i.e. $12 \mathrm{mM}$. 
Therefore, the value of measured $v_{i}$ decreases beyond pNPP maximum concentration. This behaviour is well known in the literature and it is explained by steric hindrance on the enzyme biocatalytic center most often through a non productive complex formation. In this latter case, experimental measurement of $K_{\mathrm{M}}$ is probably underestimated. [28,29,30]

Production of a non productive or inactive complex is taken into account for a new fitting procedure of experimental adsorption isotherm. In case of substrate excess, a second molecule of pNPP could be bound ALP to form an inactive complex, according to the scheme 1B.

The non competitive substrate-inhibition equation is an adapted expression of the MichaelisMenten kinetic equation resulting from the introduction of a substrate inhibition constant, $K_{\mathrm{SI}}$, as follows,

$$
-\frac{d[p N P P]}{d t}=\frac{V_{\max }^{a p p}[p N P P]}{K_{M}^{a p p}+[p N P P]+\frac{[p N P P]^{2}}{K_{S I}}}
$$

with $V_{\max }^{a p p}$ and $K_{M}^{a p p}$ are defined as Michaelis-Menten apparent constants.

In Eq. 15, $[p N P P]^{2}$ indicates that the rate tends to zero rather than $V_{\max }^{a p p}$ when $[p N P P]$ increases. The new fit procedure for the experimentally obtained isotherm, using the non competitive substrate-inhibition equation, shows a better correlation between experimental and theoretical curves. The calculated values for $V_{\max }^{a p p}, K_{M}^{a p p}$ and $K_{S I}$ were equal to $0.35 \mathrm{mM}, 46.5 \mathrm{pM} \mathrm{s}^{-1}$ and 160 , respectively. The obtained value for $K_{M}^{a p p}$ is close to that published in the literature [31,32,33]. On Fig. 4, it is interesting to note that a maximum rate was reached at a $7.5 \mathrm{mM}$ pNPP concentration determined using the following Eq. 16, concentration beyond which Michaelis-Menten equation is not totally valid.

$$
[p N P P]_{\max }=\sqrt{K_{M}^{a p p} K_{S I}}
$$


For a clear confirmation of this behaviour on the adsorption isotherm a set of experiments was performed again with other ALP concentrations and the corresponding adsorption isotherms were plotted. The variation of $\Delta R_{\mathrm{S}}$ curves for different enzyme concentrations (from 0.015 to $1.21 \mathrm{pM}$ ) against pNPP concentration (from 0.25 to $12 \mathrm{mM}$ ) versus time were recorded. Data obtained were synthesized on adsorption isotherm plots displayed in Figs. 4A-E. 

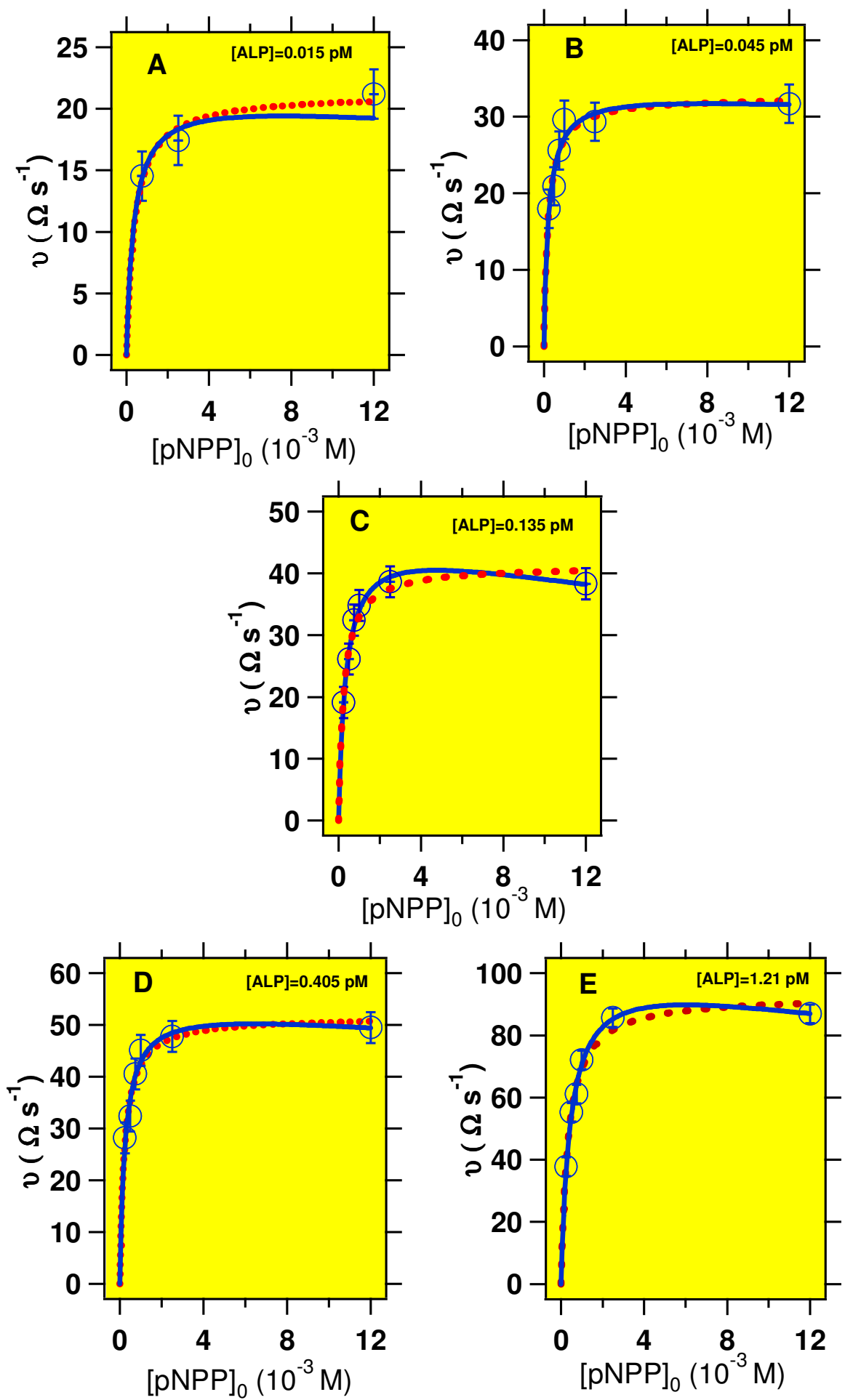

Fig. 4: Determination of adsorption isotherms with various ALP working concentrations. The maximum rate, $v_{\mathrm{i}}$-slope, is plotted against pNPP concentration. Each point represents the mean 
and the S.D. (cross-hatched bars) of set of experiments with pNPP concentration from $0.25 \mathrm{mM}$ to $12 \mathrm{mM}$ except for A. (A) $0.015 \mathrm{pM}$ as ALP working concentration. (B) $0.045 \mathrm{pM}$. (C) 0.135 pM. (D) 0.405 pM. (E) 1.21 pM. The nonlinear regressions are performed using simultaneously the Michaelis-Menten equation (dashed line, see Eq. 14 in the text) and the non competitive substrate-inhibition equation (solid line, see Eq. 15 in the text). Parameter values for each model are listed in Table 1.

The obtained parameters $\left(K_{M}, V_{\max }\right)$ determined from fitting procedure, using on the one hand the adsorption isotherm Eq. 14, and $\left(K_{M}^{a p p}, V_{\max }^{a p p}, K_{S I}\right)$, and using on the other hand Eq. 15, are listed in Table 1. The limit of detection (LOD) was observed for the isotherm displayed in Fig. 4A wherein the lowest working concentrations used with ALP and pNPP are $15 \mathrm{fM}$ and $0.75 \mathrm{mM}$, respectively.

Table 1. Parameter values obtained using the nonlinear regressions for the classical MichaelisMenten equation (Eq.14, dashed line, in Fig. 4A-E) and the non competitive substrate-inhibition equation (Eq. 15, solid line, Figs. 4A-E) with $K_{\text {SI }}$ equal to 160.

\begin{tabular}{ccccccc}
\hline [ALP] $\left(\times 10^{-12} \mathrm{M}\right)$ & 0.015 & 0.045 & 0.135 & 0.405 & 1.21 \\
\hline $\begin{array}{c}\text { [pNPP] } \\
0.25-12 \mathrm{mM}\end{array}$ & $K_{M}$ & 0.38 & 0.21 & 0.25 & 0.22 & 0.34 \\
\hline $\begin{array}{c}\text { max } \\
{[\mathrm{pNPP}]}\end{array}$ & $K_{M}^{a p p}$ & 0.36 & 0.32 & 0.35 & 0.26 & 0.39 \\
\hline $\begin{array}{c}V_{\max }^{a p p} \\
{[p N P P]_{\max }=\sqrt{K_{M}^{a p p} \times K_{S I}}}\end{array}$ & 7.60 & 7.15 & 7.50 & 7.50 & 7.90 \\
\hline
\end{tabular}


The obtained values listed in Table 1 demonstrate clearly that the intrinsic parameters, $K_{M}$ or $K_{M}^{a p p}$, are as expected, really independent of enzyme concentration, while $V_{\max }$ or $V_{\max }^{a p p}$ are dependent of ALP concentration. By considering Eq. 14 for fitting procedure, $K_{M}$ is found equal to a mean value of $0.28 \pm 0.1 \mathrm{mM}$ when $K_{M}^{a p p}$ using Eq. 15 leads to mean value of $0.33 \pm 0.06 \mathrm{mM}$. These results demonstrate that the second model is in a better agreement with the experimental results than for a classical Michaëlis-Menten approach, which involves one kind of activated complex. The substrate inhibition constant, $K_{S I}$, is found to be equal to a mean value of 160 . Inflection points for pNPP maximum concentration are extremely equal for a mean value of 7.5 mM beyond which the pNPP inhibition must be taken into account on the kinetic model.

\section{Conclusion}

The methodology here presented, based upon a direct measurement of the real component changes of electric impedance in a small channel, enables enzymatic mechanism studies with accuracy. It allows detection in small volume $(80 \mathrm{~nL})$ and still has low detection level, LOD equal to $15 \mathrm{fM}$, for bioanalytical applications. The data obtained with $\mu$-beads-ALP catalyzed hydrolysis of p-nitrophenylphosphate (pNPP) followed by the change in the microchannel resistance have demonstrated the accuracy and the sensivity of the detection scheme. Indeed, the determination of precise kinetics during ALP catalyzed hydrolysis, involving a non competitive substrate-inhibition mechanism, is the proof that this method is as sensitive as other methods without requirement of chromophore or electroactive enzyme substrate. Furthermore, the crucial advantage of the present device, with microelectrodes galvanically isolated, thus without contact with the fluidic channel, prevents the degradation of the biological sample through uncontrolled faradic reactions or unspecific adsorption on microelectrode. The results are promising not only 
for $\mu$-ELISA detection [34], but also in all experiments which require non invasive monitoring detection. Also, small dielectric PET channel associated with $\mu$-beads is a low cost equipment which offers a multi-purpose chip possibility. This fast and efficient system for the analysis of the interaction between ligands in low reaction volumes is of great interest to the community of biologists.

\section{Acknowledgments}

The authors thank Prof Hubert Girault for allowing them access to experimental facilities at the EPFL center of MicroNano Technology, Lausanne. 


\section{References}

[1] Manz, A., Graber, N., Widmer, H. M., 1990. Sens. Actuators, B. B1(1-6), 244-248.

[2] Harrison, D. J., Fluri, K., Seiler, K., Fan, Z., Effenhauser, C. S, Manz, A., 1993. Science. 261 (5123), 895-897.

[3] Emmer, A., Roeraade, J., 2005. Anal. Chim. Acta. 542(2), 137-143.

[4] Collier, A., Wang, J., Diamond, D., Dempsey, E., 2005. Anal. Chim. Acta. 550(1-2), 107-115.

[5] O'Neill, A. P., O'Brien, P., Alderman, J., Hoffman, D., McEnery, M., Murrihy, J., Glennon, J.

D., 2001. J. Chromatogr. A. 924(1-2), 259-263.

[6] Seiler, K., Harrison, D. J., Manz, A., 1993. Anal. Chem. 65 (10), 1481-1488.

[7] Crevillen, A. G., Barrigas, I., Blasco, A. J., Gonzalez, M. C., Escarpa, A., 2006. Anal. Chim. Acta. 562(2), 137-144.

[8] Wu, Z., Jensen, H., Gamby, J., Bai, X., Girault, H. H., 2004. Lab Chip. 4(5). 512-515.

[9] Chan, E. Y., Goncalves, N. M., Haeusler, R. A., Hatch, A. J., Larson, J. W., Maletta, A. M., Yantz, G. R., Carstea, E. D., Fuchs, M., Wong, G. G., 2004. Genome Res. 14(6), 1137-1146.

[10] Heleg-Shabtai, V., Gratziany, N., Liron, Z., 2006. Anal. Chim. Acta. 571(2), 228-234.

[11] Manica, D. P., Ewing, A. G., 2002. Electrophoresis. 23(21), 3735-3743.

[12] Martin, R. S., Ratzlaff, K. L., Huynh, B. H., Lunte, S. M., 2002. Anal. Chem. 74(5), 11361143.

[13] Liu, Y., Wang, H., Huang, J., Jingyu, Y., Baohong, L., Yang, P., 2009. Anal. Chim. Acta. $650(1), 77-82$.

[14] Zemann, A. J., Schnell, E., Volgger, D., Bonn, G. K., 1998. Anal. Chem. 70, 563-567.

[15] Kuban P., Hauser P. C., 2008. Anal. Chim. Acta. 607(1), 15-29. 
[16] Gamby, J., Abid, J.-P., Girault, H. H., 2005. J. Am. Chem. Soc. 127, 13300-13304.

[17] Gamby, J., Abid, J.-P., Abid, M., Ansermet, J.-P., Girault, H. H., 2006. Anal. Chem. 78, $5289-5295$.

[18] Gamby, J., Abid, J.-P., Tribollet, B., Girault, H. H., 2008. Small. 4 (6), 802-809.

[19] Gamby, J., Lazerges, M., Girault, H. H., Deslouis, C., Gabrielli, C., Perrot, H., Tribollet, B., 2008. Anal. Chem. 80, 8900-8907.

[20] Kechadi, M., Gamby, J., Chaal, L., Girault, H. H., Saidani, B., Tribollet, B. 2013. Electrochim. Acta. 105, 7-14.

[21] Faure, M., Kechadi, M., Sotta, B., Gamby, J., Tribollet, B., 2013. Electroanalysis. 25(5), $1151-1158$.

[22] Della, C. L., Bernacca, G., De Nitti, C., Massaglia, A., 1996. J. Immunol. Methods. 193(1), $51-62$.

[23] Niwa, O., Xu, Y., Halsall, H. B., Heineman, W. R., 1993. Anal. Chem. 65 (11), 1559-1563.

[24] Rossier, J. S., Girault, H. H., 2001. Lab Chip. 1, 153-157.

[25] Sarreshtedari, F., Kokabi, H., Gamby, J., Ngo, K. A., Krause, H.-J., Fardmanesh, M., 2012.

J. Electrical. Eng. 63 (7), 27-30.

[26] Avrameas, S., 1969. Immunochem. 6(1), 43-52.

[27] Gamby, F., Lazerges, M., Pernelle, C., Perrot, H., Girault, H. H., Tribollet, B., 2007. Lab. Chip. 7, 1607-1609.

[28] Morton, R. K., 1955. Biochem. J. 61, 232-240.

[29] Fernley, H. N., Walker, P. G., 1967. Biochem. J. 104, 1011-1018.

[30] Alfani, F., Gallifuoco, A., Cantarella, M., 1990. Chem. Eng. J. 43, B43-B51. 
[31] Duncan, P. H., McKneally, S. S., MacNeil, M. L., Fast, D. M., Bayse, D. D., 1984. Clin. Chem. 30(1), 93-97.

[32] Ahn, Y. S., Snow, L. D., 1993. Prep. Biochem. Biotechnol. 23(3), 409-419.

[33] Dean, R. L., 2002. Biochem. Mol. Biol. Educ., 30(6), 401-407.

[34] Henares, T. G., Funano, S.-I., Terabe, S., Mizutani, F., Sekizawa, R., Hisamoto, H., 2007. Anal. Chim. Acta. 589(2), 173-179. 